\title{
PCR and Culture Analysis of Streptococcus pneumoniae Nasopharyngeal Carriage in Healthy Children
}

\author{
Leah J. Ricketson ${ }^{1}$, Ravinder Lidder ${ }^{2}$, Robyn Thorington ${ }^{2}$, Irene Martin ${ }^{2}$, Otto G. Vanderkooi ${ }^{1,3}$, \\ Manish Sadarangani ${ }^{4,5}\left(\mathbb{D}\right.$ and James D. Kellner ${ }^{1,3, *}$ \\ 1 Department of Pediatrics, Cumming School of Medicine, University of Calgary, \\ Calgary, AB T2N 1N4, Canada; ljricket@ucalgary.ca (L.J.R.); \\ Otto.Vanderkooi@albertahealthservices.ca (O.G.V.) \\ 2 National Microbiology Laboratory, Public Health Agency of Canada, Winnipeg, MB R3E 3R2, Canada; \\ Ravinder.lidder@phac-aspc.gc.ca (R.L.); robyn.thorington@phac-aspc.gc.ca (R.T.); \\ irene.martin@phac-aspc.gc.ca (I.M.) \\ 3 Alberta Children's Hospital Research Institute, Calgary, AB T3B 6A8, Canada \\ 4 Vaccine Evaluation Center, BC Children's Hospital, Vancouver, BC V6H 3N1, Canada; \\ msadarangani@bcchr.ubc.ca \\ 5 Department of Pediatrics, University of British Columbia, Vancouver, BC V6T 1Z4, Canada \\ * Correspondence: Jim.Kellner@albertahealthservices.ca
}

check for updates

Citation: Ricketson, L.J.; Lidder, R.; Thorington, R.; Martin, I.;

Vanderkooi, O.G.; Sadarangani, M.; Kellner, J.D. PCR and Culture Analysis of Streptococcus pneumoniae Nasopharyngeal Carriage in Healthy Children. Microorganisms 2021, 9, 2116. https://doi.org/10.3390/ microorganisms 9102116

Academic Editor: Juan M. Gonzalez

Received: 15 September 2021

Accepted: 5 October 2021

Published: 8 October 2021

Publisher's Note: MDPI stays neutral with regard to jurisdictional claims in published maps and institutional affiliations.

Copyright: (c) 2021 by the authors. Licensee MDPI, Basel, Switzerland This article is an open access article distributed under the terms and conditions of the Creative Commons Attribution (CC BY) license (https:// creativecommons.org/licenses/by/ $4.0 /)$.

\begin{abstract}
Invasive Streptococcus pneumoniae disease is preceded by asymptomatic nasopharyngeal carriage. Measuring carriage in healthy populations provides data on what serotypes are present in communities, which is of interest in the era of polyvalent pneumococcal conjugate vaccines. Nasopharyngeal swabs from a survey of 682 and 800 healthy children in 2016 and 2018, respectively, were analyzed by culture and Quellung reaction to determine rates of carriage and serotypes. All swabs from 2016 and 300 randomly selected swabs from 2018 were then analyzed using real-time semi-quantitative PCR (qPCR) to detect S. pneumoniae gene targets $l y t A$, piaA, and SP2020 and determine serotype. There were $71(10.4 \%)$ and 68 (8.5\%) culture positive samples in 2016 and 2018, respectively. All of these were also positive by qPCR except one that was equivocal. In total, $46.0 \%$ of 2016 swabs were positive by qPCR. In 2018, results from the selected sample extrapolated to the complete sample showed $49.0 \%$ positive by qPCR. PCV13 serotypes were detected in $29.3 \%$ and $21.7 \%$ of S. pneumoniae qPCR positive samples from 2016 and 2018, respectively; compared with only $8.4 \%$ and $6.0 \%$ PCV13 serotypes detected by Quellung reaction in culture positive samples. Compared with culture, qPCR detected S. pneumoniae more frequently. Further, qPCR serotyping detected PCV13 serotypes in a larger proportion of samples than culture and Quellung reaction did, showing that, despite established universal childhood PCV13 immunization, vaccine serotypes can still be detected in a large proportion of young children.
\end{abstract}

Keywords: Streptococcus pneumoniae; nasopharyngeal carriage; serotype; polymerase chain reaction

\section{Introduction}

Streptococcus pneumoniae (pneumococcus) is an opportunistic pathogen because it colonizes asymptomatically, with carriage being a prerequisite for disease [1,2]. Carriage, especially in children, can act as an important reservoir that facilitates horizontal spread of S. pneumoniae throughout a community [1].

The seven-valent pneumococcal conjugate vaccine (PCV7) targets seven serotypes $(4,6 \mathrm{~B}, 9 \mathrm{~V}, 14,18 \mathrm{C}, 19 \mathrm{~F}$, and 23F) and was introduced in the province of Alberta, Canada in 2002 with three doses given before a year of age and a single booster after one year (3+ 1 schedule) [3]. In 2010, Alberta introduced the thirteen-valent pneumococcal conjugate vaccine (PCV13) with a $2+1$ schedule, which added serotypes 1, 3, 5, 6A, 7F, and 19A to the serotypes already included in the PCV7 vaccine [3]. There is a need for continual 
monitoring of the carriage of S. pneumoniae in children to understand herd immunity and serotype replacement, as well as invasive and non-invasive disease by vaccine serotypes [4]. In the city of Calgary, Alberta, after the introduction of PCVs, we have noted not only a decline in carriage of vaccine serotypes, but a decline in overall carriage of S. pneumoniae as identified by standard culture [5].

Most surveillance studies use conventional culture methods and Quellung reaction serotyping [6] for detection of S. pneumoniae from patients with non-invasive or invasive disease or nasopharyngeal carriage. However, it is recognized that some samples may be falsely reported as negative because the bacteria cannot be grown in culture [7]. This may be an issue in particular for nasopharyngeal samples where the bacterial load on a nasopharyngeal swab may be very low [8]. Polymerase chain reaction (PCR) can be used to detect the presence of pneumococcal DNA without relying on the growth of live cells in culture. This means that PCR detects a higher number of positive samples than culture methods [7,9]. Furthermore, the increased sensitivity of PCR can lead to the concurrent detection of multiple serotypes in a single person. Although detection of S. pneumoniae DNA by PCR cannot distinguish live, transmissible bacteria from non-living, non-transmissible bacterial remnants, the increased sensitivity to detect evidence of bacteria that are, or were recently, viable and transmissible, may yield more useful epidemiologic data than culture studies can. This is useful for studies evaluating whether PCV-related serotypes continue to circulate in communities with universal vaccine programs. Studies that compared the sensitivity of conventional PCR vs real-time PCR (qPCR) for the detection of $S$. pneumoniae serotypes have shown that qPCR assays are more sensitive to the presence of $S$. pneumoniae $[10,11]$.

We hypothesized that in the era of routine PCV use, children may carry a lower quantity of $S$. pneumoniae in their respiratory tracts that will be better identified using molecular methods rather than conventional culture. The purpose of this study was to investigate and understand how often $S$. pneumoniae may be detected by qPCR but not by conventional culture methods. We also compared serotyping results by Quellung reaction and qPCR.

\section{Materials and Methods}

2.1. Original Surveys to Collect Samples and Identify S. pneumoniae by Culture and Quellung Serotyping

The Calgary Area Streptococcus pneumoniae Epidemiology Research (CASPER) team conducted 13 point-prevalence surveys periodically from 2003-2018 in healthy Calgary children to determine overall and serotype-specific trends in pneumococcal nasopharyngeal carriage, identified by culture and Quellung serotyping. Results from these surveys have been previously published $[5,12]$. Nasopharyngeal samples were collected according to the methods reported previously [5,12]. Samples in this study were collected between 11 April 2016, and 2 June 2016, and between 7 May 2018, and 22 June 2018.

All subjects provided informed consent at the time of swab collection. The study was approved by the University of Calgary Conjoint Health Research Ethics Board (Ethics ID REB15-0571).

At the time of each survey, nasopharyngeal swabs were transported at an ambient temperature to the Alberta Precision Laboratories (formerly Calgary Laboratory Services) where S. pneumoniae was identified through standard culture methods using colony morphology, alpha hemolysis, optochin sensitivity, and bile solubility tests $[13,14]$. After being swabbed on culture plates, all nasopharyngeal swabs collected in 2016 and 2018 were frozen at $-85^{\circ} \mathrm{C}$ for future use. In $2016,71 / 682$ samples $(10.4 \%)$ were positive by culture for $S$. pneumoniae, and in $2018,68 / 800$ samples (8.5\%) were positive by culture.

At the time of each survey, S. pneumoniae isolates identified by culture were frozen and sent to the National Microbiology Laboratory (NML) for serotyping using the Quellung reaction with antisera obtained from SSI Diagnostica; Statens Serum Institute (Copenhagen, Denmark). More detailed methods are reported elsewhere [5,12]. 


\subsection{Identification of S. pneumoniae and Serotypes by $q P C R$}

For the current study, in 2019, all frozen nasopharyngeal samples $(n=682)$ from the 2016 carriage survey were sent to the NML for qPCR analysis. Later, in early 2020, a subset of 300 of the 800 nasopharyngeal samples from 2018 were also sent to NML. The 2018 subset included all nasopharyngeal samples that were positive by culture methods $(n=68)$ and a random selection of culture negative samples $(n=232)$. Not all samples collected in 2018 were sent for qPCR reasons due to study budget limitations. Investigators at NML were blinded about the culture results from all swabs until after testing was completed.

Target DNA was isolated using the Roche MagNA Pure 96 (Roche Diagnostics, Basel, Switzerland) automated extraction platform by processing $500 \mu \mathrm{L}$ of swab transport media with the MagNA Pure 96 DNA and Viral NA Large Volume Kit (Roche Diagnostics, Basel, Switzerland) producing a $50 \mu \mathrm{L}$ total nucleic acid eluate (as per manufacturer instruction). Extracted samples were verified for DNA isolation and integrity by PCR amplification of the Human Beta-globin endogenous gene target (internal control) [15].

Streptococcus pneumoniae qPCR detection assays were performed on the extracted DNA using the Qiagen QuantiNova SYBR Green PCR kit (Qiagen, Germantown, MA, USA) with $0.7 \mu \mathrm{M}$ primer concentration. Samples were screened for $S$. pneumoniae using qPCR targets lyt $A$ (autolysin gene) [16] and piaA (a lipoprotein component of the pneumococcal ABC transporter gene) [17]. An additional target, SP2020 (putative transcriptional regulator gene) [18], was used if the first two targets showed one negative result and one positive. A positive result of any two targets was considered positive for S. pneumoniae with no priority assigned to any particular target. An equivocal result was a single positive target. The qPCR detection primers pairings have sensitivity and specificity between $95 \%$ and 100\% [16-18]. All positive and equivocal results continued to serotype. Equivocal results that could be serotyped were considered to be positive, while not typable samples with a single positive qPCR target remained classified as equivocal (Table 1). Detection of S. pneumoniae by qPCR was performed on the ThermoFisher Scientific Applied Biosystems QuantStudio 5 Real-time PCR (ThermoFisher Scientific, Waltham, MA, USA) instrument and the cycling conditions were as follows: $95^{\circ} \mathrm{C}$ hold $2 \mathrm{~min}, 95^{\circ} \mathrm{C} 10 \mathrm{~s}$ and $60^{\circ} \mathrm{C} 30 \mathrm{~s}$ for 40 cycles, $60^{\circ} \mathrm{C}$ for $2 \mathrm{~min}$ final extension, melt curve analysis $70^{\circ} \mathrm{C}$ to $95^{\circ} \mathrm{C}$ at $0.1^{\circ} \mathrm{C}$ per second with optics.

Table 1. Assay interpretation for S. pneumoniae Detection with lyt A, piaA and SP2020, Followed by Serotyping.

\begin{tabular}{ccccc}
\hline lytA & piaA & SP2020 & Serotyping & Result \\
\hline Pos a & Pos & N/D & Serotyped or Not typed & Positive \\
Pos & Neg & Pos & Serotyped or Not typed & Positive \\
Neg & Pos & Pos & Serotyped or Not typed & Positive \\
Pos & Neg & Neg & Serotyped & Positive \\
Neg & Pos & Neg & Serotyped & Positive \\
Pos & Neg & Neg & Not Typed & Equivocal \\
Neg & Pos & Neg & Not Typed & Equivocal \\
Neg & Neg & N/D & N/D & Negative \\
\hline a Pos $=$ Positive, Neg = Negative, N/D = Not done.
\end{tabular}

Serotypes were determined using the Center for Disease Control's real-time PCR detection of 37 of 101 known pneumococcal serotypes (including all PCV13 serotypes and most other common serotypes) [10,19] using ThermoFisher Scientific TaqPath ProAmp PCR Master (ThermoFisher Scientific, Waltham, MA, USA) mix with primer and probe concentrations at $0.75 \mu \mathrm{M}$ final and $0.5 \mu \mathrm{M}$ final concentration, respectively. Table S1 details the serogroups and serotypes included in this assay, along with the fluorescent dye label used for each probe. The qPCR for serotyping was performed on ThermoFisher Scientific Applied Biosystems QuantStudio 5 Real-time PCR instrument following cycling conditions: $60^{\circ} \mathrm{C}$ pre-read $30 \mathrm{~s}$ (optics), $95^{\circ} \mathrm{C}$ polymerase activation $5 \mathrm{~min}, 95^{\circ} \mathrm{C} 15 \mathrm{~s}$ and $60{ }^{\circ} \mathrm{C} 1 \mathrm{~min}$ (optics) for 45 cycles, $60^{\circ} \mathrm{C} 1$-min post-read (optics). These conditions were optimized 
by NML throughout the process. Results were compared to conventional culture and Quellung serotyping for both 2016 and 2018 collections. A cycle threshold (CT) of $<40$ was used to indicate a positive serotype reaction $[11,20,21]$. Results that could not be serotyped by Quellung reaction are referred to as non-typeable, whereas samples that did not match one of the 37 serotypes detected by qPCR are referred to as "not typed".

\subsection{Statistical Analysis}

This study was largely a descriptive analysis, reporting on proportions of negative and positive results by culture and qPCR and proportions of serotypes found by Quellung and qPCR. For the 2018 survey, where only a random sample of 232 culture-negative swabs were assessed by qPCR, we applied the proportion of negative, equivocal, and positive PCR results to the entire sample of culture-negative results ( $n=732$ culture-negative). The results from this extrapolation were then added to the results for the 68 culture-positive swabs ( 1 equivocal, 67 positives by qPCR) to determine final numbers for Figure 1 applied to the full 2018 sample $(n=800)$.

900

800

700

600

500

400

300

200

100
$2016 n=682$

$2016 n=682$

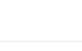
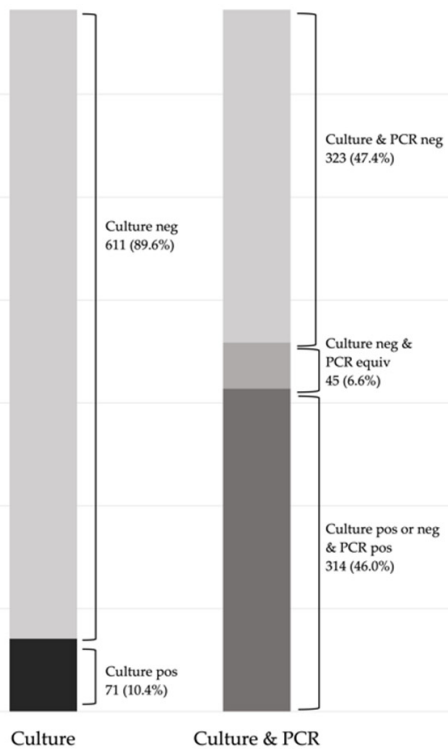

$2018 n=800$

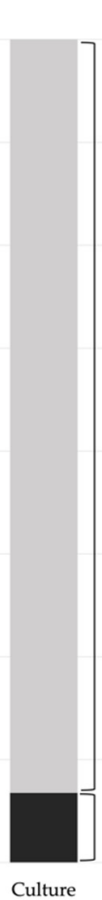

$2018 n=300$

(68 culture pos \&

232 culture neg (random)

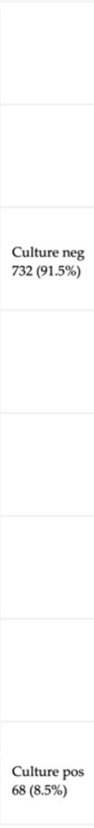

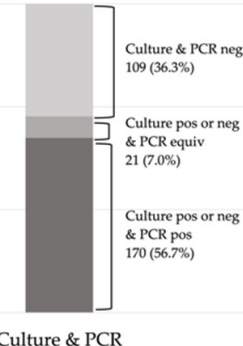

$2018 n=800$

(Extrapolated from sample of 300)

Figure 1. Culture and PCR results for 2016 and 2018, positive, negative, and equivocal.

We used a two-sample test of proportions to compare the proportion of samples from each survey that were positive for $S$. pneumoniae identified by culture and PCR in order to look for statistical significance. We also compared the proportion of serotypes (vaccine related serotypes or not) identified by Quellung and qPCR. PCV13-related serotypes that could not be distinguished from the vaccine serotype by qPCR analysis (e.g., 18C/F/B/A) were grouped with PCV13 serotypes for Tables 2 and 3. Serotypes 6C/D were also included as PCV13-related serotypes for Tables 2 and 3 due to evidence of cross-reactivity for these serotypes with PCV13 serotypes 6A/B. 
Table 2. Real-time PCR Identification of S. pneumoniae Serotypes from 314 Nasopharyngeal Samples that were qPCR Positive for S. pneumoniae, 2016 Survey.

\begin{tabular}{|c|c|c|c|}
\hline & $\begin{array}{c}\text { qPCR Results for All } \\
\text { Samples } \\
n(\%)\end{array}$ & $\begin{array}{c}\text { qPCR Results for Culture } \\
\text { Positive Samples } \\
n(\%)\end{array}$ & $\begin{array}{c}\text { qPCR Results for Culture } \\
\text { Negative Samples } \\
n(\%)\end{array}$ \\
\hline $\begin{array}{c}\text { PCV13/Related Serotype } \\
\text { Carriage }\end{array}$ & $61(19.4)$ & $9(12.7)$ & $52(21.4)$ \\
\hline NVT Carriage & $80(25.5)$ & $21(29.6)$ & $59(24.3)$ \\
\hline $\begin{array}{l}\text { Multi-Carriage by NVT and } \\
\text { PCV13-Serotypes }\end{array}$ & $31(9.9)$ & $6(8.4)$ & $25(10.3)$ \\
\hline Not Typed $b$ & $142(45.2)$ & $35(49.3)$ & $107(44.0)$ \\
\hline Total Positive by qPCR & $314(100.0)$ & $71(100.0)$ & $243(100.0)$ \\
\hline
\end{tabular}

${ }^{a}$ Due to qPCR serotyping not distinguishing between related serotype groups (e.g., 18ABCD) all serotypes related to a PCV13 serotype that could not be distinguished or have evidence of cross reactivity (e.g., 6C/D) were grouped with the PCV13 serotypes. ${ }^{b}$ The not typed could not be serotyped due to not matching one of the 37 serotypes detected by the qPCR assay.

Table 3. Real-time PCR Identification of S. pneumoniae Serotypes from 170 Nasopharyngeal Samples that were qPCR Positive for S. pneumoniae, 2018 Survey.

\begin{tabular}{|c|c|c|c|}
\hline & $\begin{array}{c}\text { qPCR Results for All } \\
\text { Samples } \\
n(\%)\end{array}$ & $\begin{array}{c}\text { qPCR Results for Culture } \\
\text { Positive Samples } \\
n(\%)\end{array}$ & $\begin{array}{c}\text { qPCR Results for Culture } \\
\text { Negative Samples } \\
n(\%)\end{array}$ \\
\hline $\begin{array}{c}\text { PCV13/Related Serotype } \\
\text { Carriage }^{\text {a }}\end{array}$ & $29(17.1)$ & $11(16.4)$ & $18(17.5)$ \\
\hline NVT Carriage & $60(35.3)$ & $29(43.3)$ & $31(30.1)$ \\
\hline $\begin{array}{c}\text { Multi-Carriage by NVT and } \\
\text { PCV13-Serotype }\end{array}$ & $8(4.7)$ & $2(3.0)$ & $6(5.8)$ \\
\hline Not Typed $b$ & $73(42.9)$ & $25(37.3)$ & $48(46.6)$ \\
\hline Total Positive by qPCR & $170(100.0)$ & $67(100.0)$ & $103(100.0)$ \\
\hline
\end{tabular}

${ }^{a}$ Due to qPCR serotyping not distinguishing between related serotype groups (e.g., 18ABCD) all serotypes related to a PCV13 serotype that could not be distinguished or have evidence of cross reactivity (e.g., 6C/D) were grouped with the PCV13 serotypes. ${ }^{b}$ The not typed could not be serotyped due to not matching one of the 37 serotypes detected by the qPCR assay.

\section{Results}

\subsection{Analysis of 2016 Samples}

The 2016 survey included nasopharyngeal swab samples from 682 children. All 71 culture-positive swabs were also qPCR positive. In addition, 243/611 culture-negative swabs were qPCR positive and $45 / 611$ of culture-negative swabs were equivocal by qPCR (Figure 1 ). Thus, a total of $314 / 682$ total samples $(46.0 \%)$ were qPCR positive and a $45 / 682$ total samples $(6.6 \%)$ were qPCR equivocal. There was a significantly higher number positive by qPCR than by culture $(46.0 \%$ vs $10.4 \%, p<0.001)$.

Real-time PCR serotyping results from 2016 are shown in Table 2. By Quellung reaction, $6(8.4 \%)$ of the 71 culture-positive samples had PCV13-related serotypes, while $64(90.1 \%)$ were non-vaccine serotypes (NVT) and $1(1.4 \%)$ was non-typeable. From the 314 qPCR positive samples, a single serotype was identified by qPCR for $135(43.0 \%)$ samples, while 2 or more serotypes were identified in $37(11.8 \%)$ samples and $142(45.2 \%)$ could not be serotyped due to the limited number of serotypes in the qPCR assay. In total, 92/314 (29.3\%) PCR positive samples had 1 or more PCV13 serotypes compared to $8.4 \%$ culture positive samples that had PCV13 serotypes by Quellung reaction $(p<0.001)$.

The serotype identified by Quellung was not identified by qPCR in 14 of 71 culture positive samples. In one sample there was a mismatch with serotype 19A identified by Quellung and qPCR could not identify a serotype. For the other 13, the serotype found by Quellung was a serotype that could not be detected by qPCR as it was not one of the 37 serotypes included in the assay. 


\subsection{Analysis of 2018 Samples}

The 2018 survey included 800 children. All 68 culture-positive nasopharyngeal swab samples were also qPCR positive except one sample that yielded an equivocal qPCR result. A random selection of 232/732 culture negative swabs were also selected for qPCR analysis, for a total of 300 samples. The qPCR results from the culture-negative swabs $(n=232)$ were extrapolated to apply to the entire sample of culture-negative swabs $(n=732)$. There were 103/232 culture-negative swabs that were qPCR positive and 20/232 culture-negative swabs that were equivocal by qPCR. The extrapolated total of culture-negative swabs that were estimated to be qPCR positive for $S$. pneumoniae, combined with the 68 culturepositive, was 392/800 (49.0\%) and 64/800 (8.0\%) with equivocal results (Figure 1$)$. There was a significantly higher number estimated to be positive by qPCR than by culture $(49.0 \%$ vs. $8.5 \%, p<0.001)$.

Serotyping results from 2018 are shown in Table 3. By Quellung reaction, 4 (6.0\%) of the culture-positive samples had PCV13-related serotypes, while $63(94.0 \%)$ were NVT, 0 were non-typeable and 1 could not be serotyped as it was non-viable after storage. From the 170 PCR positive samples for 2018, a single serotype was identified by qPCR for $74(43.5 \%)$ samples, while 2 or more serotypes were identified for $23(13.5 \%)$ and $73(42.9 \%)$ could not be serotyped due to limitation of the assay. In total, 37/170 (21.7\%) samples had 1 or more PCV13 serotypes, which was significantly more than found by Quellung $(p<0.001)$.

The serotype identified by Quellung was not identified by qPCR for 17 of 68 culture positive samples. As with the 2016 samples, the serotype found by culture was a serotype that could not be detected by qPCR as it was not one of the 37 serotypes included in the assay.

Table S2 shows the isolates with Quellung reaction identified serotypes which are not included in the molecular assay, but also had additional qPCR detected serotypes for both years. For 30 of these 31 samples, 2 or more serotypes were identified by the combination of Quellung and PCR, which would suggest co-carriage. Quellung was repeated on all of these samples, confirming the original serotype results.

\section{Discussion}

In the era of established PCV use, it is important to understand how well vaccination is controlling disease and asymptomatic nasopharyngeal carriage caused by vaccine serotypes, as well as the overall level of S. pneumoniae carriage. In the current study, we found that qPCR detected S. pneumoniae more frequently than conventional culture in nasopharyngeal samples obtained from healthy children, long after the introduction of PCVs in Canada and after we started to observe reduced overall S. pneumoniae carriage using standard culture methods [5]. Further, qPCR serotyping detected PCV13 serotypes in a larger proportion of S. pneumoniae-positive samples than did Quellung serotyping. It is important to note that the nasopharyngeal samples collected in our surveys were from healthy children and collected for research and surveillance purposes only. Thus, it was beyond the scope of our study to determine whether each strain of S. pneumoniae that was identified by culture or qPCR was transmissible. However, our findings do suggest that PCV13 serotype strains of S. pneumoniae are still commonly identified in our population by qPCR, if not by culture, from asymptomatic carriage samples. Concurrent studies of clinical samples from cases of invasive pneumococcal disease (IPD) in Canada have identified that PCV13 serotypes are causing 26\% of all cases of IPD in children and 31\% of IPD in all ages during the same time period as these surveys $[22,23]$. Thus, the current findings, taken with previously reported findings on IPD serotypes, indicate that although universal use of childhood PCVs has reduced vaccine serotype invasive disease, vaccine serotypes have not been eliminated [23].

Other studies have compared the quantitative load of S. pneumoniae in nasopharyngeal samples collected from sick patients diagnosed with bacteremic pneumococcal infections. For example, Messaoudi et al. found that the nasopharyngeal concentration of serotypes 
also found in clinical blood cultures was 3 log-fold higher than serotypes found only in the nasopharynx and not in blood [24].

The CASPER team has conducted 13 point-prevalence studies measuring nasopharyngeal carriage, using conventional culture methods in convenience samples of healthy young children during routine immunization visits at public health centers in Calgary since $2003[5,12]$. In addition to sharp declines in vaccine-serotype carriage, overall S. pneumoniae carriage declined following widespread use of PCVs. In Calgary, PCV7 was introduced in 2002 and PCV13 was introduced in 2010. Prior to 2010, the overall carriage rate was $19.9 \%$, but in 2010-2012, nasopharyngeal carriage declined to 13.2\% [5]. In 2016 and 2018, the overall carriage rates were further reduced to $10.6 \%$ and $8.6 \%$, respectively. The current study compares and bridges culture and qPCR results from the two most recent surveys, which was enabled by freezing and retaining nasopharyngeal swabs first used for culture and later for qPCR. Swabs collected from prior surveys were not retained and so no further comparisons can be made to determine if the differences in detection of S. pneumoniae by culture and qPCR would have been as large in previous years in which culture detection was higher.

While numerous other studies of S. pneumoniae carriage in children in the PCV era have reported no change in overall carriage levels (due to replacement of vaccine serotype strains with non-vaccine serotype strains), there is also evidence from clinical trials and other observational studies of reduced overall carriage similar to what we have reported $[25,26]$.

The targets used for PCR detection of S. pneumoniae have evolved over time $[27,28]$. The current method of choice for detection of S. pneumoniae by qPCR is lytA (autolysin gene) [16]. The lytA primer and probe used in this study were designed by da Gloria Carvalho et al. to accurately differentiate S. pneumoniae from other closely related Streptococcus mitis group species [16]. The second detection target used in this study is the pia $A$ (a lipoprotein component of the pneumococcal $\mathrm{ABC}$ transporter gene), which is used to increase the specificity of pneumococcal identification, although this target has been reported as being absent from some non-encapsulated pneumococci [17]. A recent report described an additional target, SP2020 (putative transcriptional regulator gene) [18], used along with $l y t A$ as a powerful strategy for the identification of S. pneumoniae. In this study, all three targets (lyt A, piaA and SP2020) were used, but there was no priority assigned to any targets and positive or equivocal categorization was based on any combination of targets as well as serotyping results (Table 1). Given that each of the primers used have very high but not $100 \%$ sensitivity to detect $S$. pneumoniae, it is expected that by chance, only a single primer will be positive in some samples, leading to the equivocal designation if no serotype was identified.

Samples from each survey were analyzed by qPCR for S. pneumoniae detection and serotyping on separate occasions at the NML and the methods were refined on each occasion. The Centers for Disease Control's S. pneumoniae molecular serotyping assay [10] was adapted and optimized. The assay detects 37 serogroups/serotypes, which are either included in the PCV13 vaccine or are otherwise common serotypes causing pneumococcal disease [10]. As a result, current qPCR serotyping assays are not as complete as Quellung reaction serotyping, since not all known serotypes are identified. Samples that are nontypeable with the Quellung reaction indicates that a cultured S. pneumoniae strain is not one of 101 known serotypes $[19,29,30]$. Conversely, samples that are not typed with qPCR serotyping would be expected to most often still have a known serotype that is not one of the 37 identified by the qPCR assay. Further, qPCR serotyping in some cases only detects groups of serotypes e.g., serotypes 18CFBA, whereas Quellung reaction can distinguish between each of the individual serotypes: $18 \mathrm{C} / 18 \mathrm{~F} / 18 \mathrm{~B} / 18 \mathrm{~A}$.

In this study, when qPCR serotyping results detected a serotype group which included a PCV13 serotype e.g., 18C/18F/18B/18A (of which 18C is PCV13 serotype), we designated it as a PCV13 serotype. Thus, there may be an overestimate of the proportion of samples with vaccine serotypes if the actual serotype for these samples was non-PCV13. However, 
this only applied to a small number of samples with limited serotypes (6A/B/C/D, 9V/A, and $18 \mathrm{C} / \mathrm{F} / \mathrm{B} / \mathrm{A}$ ) and the non-PCV13 serotype(s) in these groups are rare.

There was just one discrepancy in the serotypes determined by the Quellung reaction and qPCR, with a sample from 2016. The sample was positive by culture and qPCR but not typed by qPCR serotyping although the serotype found by Quellung (19A) was included in the qPCR assay, and therefore should have been detectable by qPCR. The Quellung was repeated for this sample confirming the 19A result. It is possible that in the clinical sample, the concentration of the DNA was too low to be detected by the qPCR. In one study that compared PCR and Quellung serotyping, 19A was frequently involved in discordant results [31]. When Quellung reactions were repeated, the Quellung result often changed to match the PCR result, while the PCR results were the same on repeat analysis, suggesting errors with the Quellung reaction may be more common [31]. Thirty other samples in this study had a different serotype found by qPCR than by Quellung, but the Quellung serotype was a serotype not included in the qPCR assay; therefore, it could not have been identified by qPCR. We did not consider these to be discrepant serotypes, but rather evidence of co-carriage by the serotype identified by Quellung and the one identified by qPCR.

PCV13-related serotypes were found more often by qPCR than by culture, suggesting vaccination may have reduced the quantitative load of bacteria with these serotypes, but not eliminated them. Of particular interest was the presence of serotype 4 in $3.5 \%$ of the samples by qPCR but $0 \%$ in culture-positive samples. There has been a recent outbreak of serotype 4 among adults in Calgary, especially homeless persons [32]. The findings from the current study suggest that children may still be an important reservoir for serotype 4 . Similarly, other PCV13 serotypes, including 3 and 5, and to a lesser degree, serotypes 7F and 1, were not identified by culture/Quellung analysis but were frequently found by qPCR. Serotype 3 and 7F also continue to cause high levels of disease throughout Canada despite widespread vaccination [23]. In contrast, serotype 19A, which is also a vaccine type that continues to cause disease in Canada, was not commonly found in carriage by PCR or by culture (Table S3).

Serotypes 11A, 22F, 15A, 16F, and 23A were common in carriage but are not included in current PCVs, although $11 \mathrm{~A}$ and $22 \mathrm{~F}$ are included in the 23-valent pneumococcal polysaccharide vaccine [33]. All of these serotypes are among the more common serotypes causing disease in Canada between 2014 and 2018 [23]. Serotype 33F was less common but also present by qPCR. Serotypes 22F and 33F are included in a new 15-valent PCV [34] and they are also included along with serotypes $8,10 \mathrm{~A}, 11 \mathrm{~A}, 12 \mathrm{~F}$, and $15 \mathrm{~B}$ in a new 20 -valent PCV [35].

\section{Conclusions}

Compared with culture, qPCR detected S. pneumoniae far more frequently in nasopharyngeal samples obtained from healthy children. In addition, qPCR serotyping detected PCV13 serotypes in a higher proportion of samples with S. pneumoniae than Quellung reaction serotyping did, showing that, despite established universal childhood PCV13 immunization, asymptomatic carriage of vaccine serotypes has not declined to the very low levels indicated by studies using culture and Quellung reaction serotyping only. Future studies of S. pneumoniae carriage may preferentially use PCR detection and serotyping in place of culture and Quellung techniques, particularly if more serotypes can be added to the existing PCR assays.

Supplementary Materials: The following are available online at https: / www.mdpi.com/article / 10.3390/microorganisms9102116/s1, Table S1: The 37 Serotypes Detected by the CDC's S. pneumoniae qPCR Assay and Fluorescent Dye Associated with Each Probe, Table S2: Detection of Additional Serotypes by qPCR in Samples with Quellung Results Which Are Not Included in the 37-Serotype Molecular Assay, Table S3: Serotype Prevalence in Nasopharyngeal Samples Sent to NML, 2016 and 2018 combined. Samples had up to 4 serotypes Co-Carriage, All Serotypes are Counted. 
Author Contributions: Conceptualization, J.D.K. methodology, J.D.K., L.J.R., I.M., R.L. and R.T.; validation, R.L., I.M. and R.T.; formal analysis, L.J.R., J.D.K., M.S., I.M., R.L. and R.T.; investigation, I.M., R.L., R.T.; resources, J.D.K. and I.M.; data curation, L.J.R., R.T., R.L.; writing-original draft preparation, L.J.R. and J.D.K.; writing-review and editing, L.J.R., J.D.K., I.M., R.L., R.T., O.G.V., M.S.; visualization, L.J.R. and J.D.K.; supervision, J.D.K.; project administration, J.D.K. and I.M.; funding acquisition, J.D.K. and O.G.V. All authors have read and agreed to the published version of the manuscript.

Funding: This research was funded, in part, by an unrestricted grant-in-aid from Pfizer Canada (Grant \#WI222857), for the CASPER pneumococcal surveillance study.

Institutional Review Board Statement: The study was conducted according to the guidelines of the Declaration of Helsinki, and approved by the University of Calgary and Alberta Health Services Conjoint Health Research Ethics Board (Ethics ID REB15-0571, 1 April 2000).

Informed Consent Statement: Informed consent was obtained from all subjects involved in the study.

Data Availability Statement: The dataset for this study will be available in the University of Calgary PRISM Repository.

Acknowledgments: The authors would like to acknowledge the research staff and students at National Microbiology Lab, Winnipeg. We would also like to acknowledge the contributions in sample and data collection and consolidation by the ACHIEVE research team (Shannon Pyra, Joslyn Gray, Nicole MacMillan), and the Alberta Precision Laboratories (Tracie Llyod).

Conflicts of Interest: The authors declare no conflict of interest. The funders had no role in the design of the study; in the collection, analyses, or interpretation of data; in the writing of the manuscript, or in the decision to publish the results.

\section{References}

1. Bogaert, D.; de Groot, R.; Hermans, P.W.M. Streptococcus pneumoniae colonisation: The key to pneumococcal disease. Lancet Infect. Dis. 2004, 4, 144-154. [CrossRef]

2. Gray, B.M.; Converse, G.M., 3rd; Dillon, H.C., Jr. Epidemiologic studies of Streptococcus pneumoniae in infants: Acquisition, carriage, and infection during the first 24 months of life. J. Infect. Dis. 1980, 142, 923-933. [CrossRef]

3. Public Health Agency of Canada. Update on the Use of Pneumococcal Vaccines in Adults 65 Years of Age and Older-A Public Health Perspective: An Advisory Committee Statement (ACS) National Advisory Committee on Immunization (NACI). Available online: https:/ / www.canada.ca/en/public-health/services/publications/healthy-living/update-on-the-use-of-pneumococcalvaccines-in-adult.html (accessed on 13 March 2021).

4. $\quad$ LeBlanc, J.J.; ElSherif, M.; Ye, L.; MacKinnon-Cameron, D.; Ambrose, A.; Hatchette, T.F.; Lang, A.L.S.; Gillis, H.D.; Martin, I.; Demczuk, W.; et al. Streptococcus pneumoniae serotype 3 is masking PCV13-mediated herd immunity in Canadian adults hospitalized with community acquired pneumonia: A study from the Serious Outcomes Surveillance (SOS) Network of the Canadian immunization research Network (CIRN). Vaccine 2019, 37, 5466-5473. [CrossRef]

5. Ricketson, L.J.; Wood, M.L.; Vanderkooi, O.G.; MacDonald, J.C.; Martin, I.E.; Demczuk, W.H.; Kellner, J.D. Trends in asymptomatic nasopharyngeal colonization with S. pneumoniae after introduction of the 13-valent pneumococcal conjugate vaccine in Calgary, Canada. Pediatr. Infect. Dis. J. 2014, 33, 724-730. [CrossRef] [PubMed]

6. Austrian, R. The quellung reaction, a neglected microbiologic technique. Mt. Sinai J. Med. N. Y. 1976, 43, 699-709.

7. Da Gloria Carvalho, M.; Pimenta, F.C.; Jackson, D.; Roundtree, A.; Ahmad, Y.; Millar, E.V.; O’Brien, K.L.; Whitney, C.G.; Cohen, A.L.; Beall, B.W. Revisiting pneumococcal carriage by use of broth enrichment and PCR techniques for enhanced detection of carriage and serotypes. J. Clin. Microbiol. 2010, 48, 1611-1618. [CrossRef] [PubMed]

8. Hamer, D.H.; Egas, J.; Estrella, B.; William, B.M.; Jeffrey, K.G.; Sempértegui, F. Assessment of the Binax NOW Streptococcus pneumoniae Urinary Antigen Test in Children with Nasopharyngeal Pneumococcal Carriaged. Clin. Infect. Dis. 2002, 34, $1025-1028$. [CrossRef]

9. Saukkoriipi, A.; Leskela, K.; Herva, E.; Leinonen, M. Streptococcus pneumoniae in nasopharyngeal secretions of healthy children: Comparison of real-time PCR and culture from STGG-transport medium. Mol. Cell Probes. 2004, 18, 147-153. [CrossRef]

10. Pimenta, F.C.; Roundtree, A.; Soysal, A.; Bakir, M.; du Plessis, M.; Wolter, N.; von Gottberg, A.; McGee, L.; Carvalho Mda, G.; Beall, B. Sequential triplex real-time PCR assay for detecting 21 pneumococcal capsular serotypes that account for a high global disease burden. J. Clin. Microbiol. 2013, 51, 647-652. [CrossRef]

11. Azzari, C.; Moriondo, M.; Indolfi, G.; Cortimiglia, M.; Canessa, C.; Becciolini, L.; Lippi, F.; de Martino, M.; Resti, M. Realtime PCR Is More Sensitive than Multiplex PCR for Diagnosis and Serotyping in Children with Culture Negative Pneumococcal Invasive Disease. PLoS ONE 2010, 5, e9282. [CrossRef] 
12. Kellner, J.D.; Scheifele, D.; Vanderkooi, O.G.; Macdonald, J.; Church, D.L.; Tyrrell, G.J. Effects of routine infant vaccination with the 7-valent pneumococcal conjugate vaccine on nasopharyngeal colonization with S. pneumoniae in children in Calgary, Canada. Pediatr. Infect. Dis. J. 2008, 27, 526-532. [CrossRef] [PubMed]

13. Becker, K.; Skov, R.L.; von Eiff, C. Staphylococcus, Micrococcus, and Other Catalase-Positive Cocci. In Manuel of Clinical Microbiology, 11th ed.; Jorgensen, J.H., Carroll, K.C., Funke, G., Pfaller, M.A., Landry, M.L., Richter, S.S., Warnock, D.W., Eds.; American Society for Microbiology Press: Washington, DC, USA, 2015; Volume 1, pp. 354-382.

14. Christensen, J.J.; Ruoff, K.L. Aerococcus, Abiotrophia, and Other Aerobic Catalase-Negative, Gram Positive Cocci. In Manual of Clinical Microbiology; Jorgensen, J.H., Carroll, K.C., Funke, G., Pfaller, M.A., Landry, M.L., Richter, S.S., Warnock, D.W., Eds.; American Society for Microbiology Press: Washington, DC, USA, 2015; Volume 1, pp. 422-436.

15. Lingappa, J.R.; Lawrence, W.; West-Keefe, S.; Gautom, R.; Cookson, B.T. Diagnosis of community-acquired pertussis infection: Comparison of both culture and fluorescent-antibody assays with PCR detection using electrophoresis or dot blot hybridization. J. Clin. Microbiol. 2002, 40, 2908-2912. [CrossRef] [PubMed]

16. Da Gloria Carvalho, M.; Tondella, M.L.; McCaustland, K.; Weidlich, L.; McGee, L.; Mayer, L.W.; Steigerwalt, A.; Whaley, M.; Facklam, R.R.; Fields, B.; et al. Evaluation and Improvement of Real-Time PCR Assays Targeting lyt $A$, ply, and psaA Genes for Detection of Pneumococcal DNA. J. Clin. Microbiol. 2007, 45, 2460. [CrossRef] [PubMed]

17. Trzcinski, K.; Bogaert, D.; Wyllie, A.; Chu, M.L.; van der Ende, A.; Bruin, J.P.; van den Dobbelsteen, G.; Veenhoven, R.H.; Sanders, E.A. Superiority of trans-oral over trans-nasal sampling in detecting Streptococcus pneumoniae colonization in adults. PLoS ONE 2013, 8, e60520. [CrossRef]

18. Tavares, D.A.; Handem, S.; Carvalho, R.J.; Paulo, A.C.; de Lencastre, H.; Hinds, J.; Sa-Leao, R. Identification of Streptococcus pneumoniae by a real-time PCR assay targeting SP2020. Sci. Rep. 2019, 9, 3285. [CrossRef] [PubMed]

19. Ganaie, F.; Maruhn, K.; Li, C.; Porambo, R.J.; Elverdal, P.L.; Abeygunwardana, C.; van der Linden, M.; Duus, J.; Sheppard, C.L.; Nahm, M.H. Structural, Genetic, and Serological Elucidation of Streptococcus pneumoniae Serogroup 24 Serotypes: Discovery of a New Serotype, 24C, with a Variable Capsule Structure. J. Clin. Microbiol. 2021, 59, e0054021. [CrossRef] [PubMed]

20. Wyllie, A.L.; Rümke, L.W.; Arp, K.; Bosch, A.A.T.M.; Bruin, J.P.; Rots, N.Y.; Wijmenga-Monsuur, A.J.; Sanders, E.A.M.; Trzciński, K. Molecular surveillance on Streptococcus pneumoniae carriage in non-elderly adults; little evidence for pneumococcal circulation independent from the reservoir in children. Sci. Rep. 2016, 6, 34888. [CrossRef]

21. Pasinato, A.; Indolfi, G.; Marchisio, P.; Valleriani, C.; Cortimiglia, M.; Spanevello, V.; Chiamenti, G.; Buzzetti, R.; Resti, M.; Azzari, C. Pneumococcal serotype distribution in 1315 nasopharyngeal swabs from a highly vaccinated cohort of Italian children as detected by RT-PCR. Vaccine 2014, 32, 1375-1381. [CrossRef]

22. Ricketson, L.J.; Kellner, J.D. Invasive Pneumococcal Disease (IPD) Trends 1998 to Mid-2019 in Calgary, Canada: An Interrupted Time Series Analysis. In Proceedings of the International Syposium on Pneumococcus and Pneumococcal Disease, Toronto, ON, Canada, 19-23 June 2020; Abstract 104. Available online: https://cslide.ctimeetingtech.com/isppd20/attendee/confcal/session/ calendar?q=Ricketson (accessed on 25 September 2020).

23. Demczuk, W.; Griffith, A.; Montes, K.; Mallari, R.; Thorington, R.; Sawatzky, P.; Martin, I.; Mulvey, M. Laboratory Surveillance of Invasive Streptococcal Disease in Canada Annual Report 2018; Public Health Agency of Canada: Ottawa, ON, Canada, 2018. Available online: https:/ / publications.gc.ca/collections/collection_2021/aspc-phac/HP57-4-2018-eng.pdf (accessed on 3 September 2021).

24. Messaoudi, M.; Milenkov, M.; Albrich, W.C.; van der Linden, M.P.; Bénet, T.; Chou, M.; Sylla, M.; Barreto Costa, P.; Richard, N.; Klugman, K.P.; et al. The Relevance of a Novel Quantitative Assay to Detect up to 40 Major Streptococcus pneumoniae Serotypes Directly in Clinical Nasopharyngeal and Blood Specimens. PLoS ONE 2016, 11, e0151428. [CrossRef]

25. Van Gils, E.J.; Veenhoven, R.H.; Hak, E.; Rodenburg, G.D.; Bogaert, D.; Ijzerman, E.P.; Bruin, J.P.; van Alphen, L.; Sanders, E.A. Effect of reduced-dose schedules with 7-valent pneumococcal conjugate vaccine on nasopharyngeal pneumococcal carriage in children: A randomized controlled trial. JAMA 2009, 302, 159-167. [CrossRef]

26. Løvlie, A.; Vestrheim, D.F.; Aaberge, I.S.; Steens, A. Changes in pneumococcal carriage prevalence and factors associated with carriage in Norwegian children, four years after introduction of PCV13. BMC Infect. Dis. 2020, 20, 29. [CrossRef]

27. Johnsborg, O.; Eldholm, V.; Bjørnstad, M.L.; Håvarstein, L.S. A predatory mechanism dramatically increases the efficiency of lateral gene transfer in Streptococcus pneumoniae and related commensal species. Mol. Microbiol. 2008, 69, 245-253. [CrossRef]

28. Suzuki, N.; Yuyama, M.; Maeda, S.; Ogawa, H.; Mashiko, K.; Kiyoura, Y. Genotypic identification of presumptive S. pneumoniae by PCR using four genes highly specific for S. pneumoniae. J. Med. Microbiol. 2006, 55, 709-714. [CrossRef] [PubMed]

29. Hanage, W.P.; Kaijalainen, T.; Saukkoriipi, A.; Rickcord, J.L.; Spratt, B.G. A successful, diverse disease-associated lineage of nontypeable pneumococci that has lost the capsular biosynthesis locus. J. Clin. Microbiol. 2006, 44, 743-749. [CrossRef] [PubMed]

30. Shayegani, M.; Parsons, L.M.; Gibbons, W.E., Jr.; Campbell, D. Characterization of nontypable Streptococcus pneumoniae-like organisms isolated from outbreaks of conjunctivitis. J. Clin. Microbiol. 1982, 16, 8-14. [CrossRef] [PubMed]

31. Richter, S.S.; Heilmann, K.P.; Dohrn, C.L.; Riahi, F.; Diekema, D.J.; Doern, G.V. Evaluation of pneumococcal serotyping by multiplex PCR and quellung reactions. J. Clin. Microbiol. 2013, 51, 4193-4195. [CrossRef] [PubMed]

32. Kellner, J.D.; Ricketson, L.J.; Demczuk, W.H.B.; Martin, I.; Tyrrell, G.J.; Vanderkooi, O.G.; Mulvey, M.R. Whole-Genome Analysis of Streptococcus pneumoniae Serotype 4 Causing Outbreak of Invasive Pneumococcal Disease, Alberta, Canada. Emerg. Infect. Dis. 2021, 27, 1867-1875. [CrossRef] 
33. Latifi-Navid, H.; Latifi-Navid, S.; Mostafaiy, B.; Jamalkandi, S.A.; Ahmadi, A. Pneumococcal Disease and the Effectiveness of the PPV23 Vaccine in Adults: A Two-Stage Bayesian Meta-Analysis of Observational and RCT Reports. Sci. Rep. 2018, 8, 11051. [CrossRef] [PubMed]

34. Stacey, H.L.; Rosen, J.; Peterson, J.T.; Williams-Diaz, A.; Gakhar, V.; Sterling, T.M.; Acosta, C.J.; Nolan, K.M.; Li, J.; Pedley, A.; et al. Safety and immunogenicity of 15-valent pneumococcal conjugate vaccine (PCV-15) compared to PCV-13 in healthy older adults. Hum. Vaccines Immunother. 2019, 15, 530-539. [CrossRef]

35. Hurley, D.; Griffin, C.; Young, M.; Scott, D.A.; Pride, M.W.; Scully, I.L.; Ginis, J.; Severs, J.; Jansen, K.U.; Gruber, W.C.; et al. Safety, Tolerability, and Immunogenicity of a 20-Valent Pneumococcal Conjugate Vaccine (PCV20) in Adults 60 to 64 Years of Age. Clin. Infect. Dis. 2021, 73, e1489-e1497. [CrossRef] 